\title{
Griscelli Syndrome Type 2: Long-Term Follow-Up after Unrelated Donor Bone Marrow Transplantation
}

\author{
Alfredo Rossi ${ }^{a}$ Riccardo G. Borroni ${ }^{d}$ Anna Maria Carrozzo ${ }^{b}$ Catia de Felice ${ }^{b}$ \\ Adriana Menichelli $^{c}$ Marta Carlesimo $^{a}$ Stefano Calvieri ${ }^{a}$ \\ ${ }^{a}$ Department of Dermatology, La Sapienza University, and Departments of ${ }^{b}$ Dermatology and ${ }^{c}$ Public Health \\ and Cell Biology, Tor Vergata University, Rome, and ${ }^{\mathrm{d}}$ Department of Dermatology, University of Modena \\ and Reggio Emilia, Modena, Italy
}

\section{Key Words}

Griscelli syndrome $\cdot$ Silvery hair .

Partial albinism $\cdot$ Hemophagogytic lymphohistiocytosis - Bone marrow transplantation

\begin{abstract}
Griscelli syndrome (GS) is a rare autosomal recessive disease characterized by silvery hair ('partial albinism'). Three forms exist; GS type 2 (GS2), the most common one, is characterized by severe primary immunodeficiency with acute episodes of hemophagocytic lymphohistiocytosis (HLH) which may be fatal in the absence of hematopoietic stem cell transplantation. A 5-year-old boy with HLH was referred to us because of silvery-gray hair present since birth. Abnormal pigment clumps were observed in the medulla of hair shafts on light microscopy. Electron microscopy of a skin biopsy revealed melanosomes in melanocytes, but not in keratinocytes. Leukocytes were devoid of intracytoplasmic granules on blood smear. Neurological signs were absent. Genotyping revealed a homozygous haplotype for polymorphic markers linked to the RAB27A locus, but no RAB27A mutation. A diagnosis of GS2 was established. The patient received bone marrow transplantation (BMT) from an
\end{abstract}

unrelated donor, and after 72 months he did not show relapse of $\mathrm{HLH}$. The long, uneventful follow-up supports the use of BMT from an unrelated donor if transplantation from a relative is not possible.

Copyright $\odot 2009$ S. Karger AG, Basel

\section{Introduction}

Griscelli syndrome (GS, OMIM 214450), first described in 1978 [1], is a rare autosomal recessive disease characterized clinically by hypopigmentation of the scalp hair, eyebrows and eyelashes (silvery hair or 'partial albinism') and microscopically by the presence of large clumps of pigment in hair shafts, with accumulation of melanosomes in melanocytes. Based on associated clinical features and the underlying genetic defect, 3 mutually exclusive forms have been recognized. All of them share a defect in the transfer of melanosomes from melanocytes to keratinocytes [2]. GS type 1, which likely corresponds to Elejalde syndrome (ES) or melanolysosomal neurocutaneous syndrome, is also characterized by primary central nervous system (CNS) dysfunction and is caused by mutations in the myosin Va (MYO5A) gene. GS type 2 (GS2), the most common type, is associated with severe primary immunodeficiency causing 'accelerated phases' of hemophagocytic lymphohistiocytosis (HLH) [1]. This consists in a macrophage and T-lymphocyte activation syndrome, often triggered by infections, characterized by hepatosplenomegaly, fever, pancytopenia, hemorrhages and elevated levels of transaminases and triglycerides. Neurological involvement secondary to CNS infiltration by $\mathrm{T}$ cells and macrophages can be present. Death generally occurs if the patient does not receive hematopoietic stem cell transplantation (HSCT). GS2 is caused by homozygous mutations of the RAB27A gene [3]. Rab27a protein regulates the exocytosis of secretory lysosomes such as melanosomes in melanocytes and lytic granules in T lymphocytes [4]. Patients with GS type 3 show hypopigmentation only and carry mutations of the melanophilin $(M L P H)$ gene. The protein complex formed by Rab27a, Mlph, and MyoVa has recently been shown to be essential for the capture and local movement of melanosomes in the actinrich cell periphery of melanocytes [5].

Other inherited syndromes are characterized by silvery hair. Chediak-Higashi syndrome (CHS) shares with GS both hypopigmented hair and HLH. In contrast to

\section{KARGER}

Fax +41613061234 E-Mail karger@karger.ch www.karger.com
Riccardo G. Borroni, MD

University of Modena and Reggio Emilia

Department of Dermatology, Via del Pozzo, 71

IT-41100 Modena (Italy)

Tel. +39 059422 2812, Fax +39 059422 4271, E-Mail riccardoborroni@gmail.com 


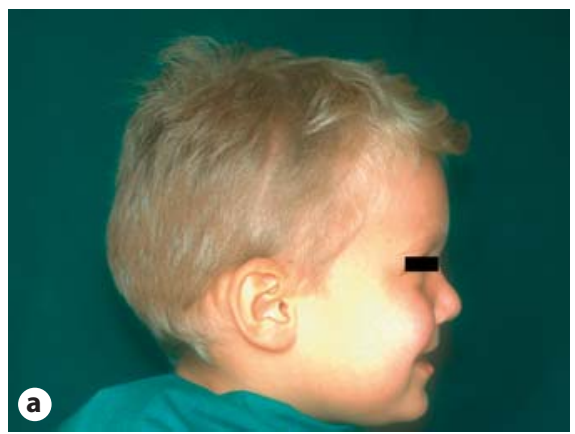

Fig. 1. a Typical silvery-gray appearance of hair. b Light microscopy of hair shafts showing pigment clusters irregularly disposed in the medulla and in the cortex. c Skin biopsy showing melanosomes in a melanocyte but scantly pigmented keratinocytes in the basal layer. d Histological cross-section of a hair follicle showing pigment deposits in the medulla and in the cortex of a hair shaft. e Light microscopy of normal hair shafts from a normal individual showing regular distribution of melanin.

GS, CHS typically displays abnormal giant cytoplasmic inclusions in leukocytes, regular distribution of melanin clumps in the hair shaft, and giant melanosomes in both melanocytes and keratinocytes. Although the definitive distinction between GS and $\mathrm{CHS}$ relies on genetic analysis, simple microscopic features are helpful in establishing a prompt diagnosis of GS2.

\section{Case Report}

A 5-year-old boy who was hospitalized at the Department of Pediatrics for recurrent fever was referred to our Department of Dermatology in February 2001 because of gray hair that had been present since birth. He was the only child of unrelated parents. On physical examination, silverygray hair, eyebrows and eyelashes were evident (fig. 1a). A chest X-ray showed a small density in the anterior basal part of the right lung. Abdominal ultrasound revealed hepatosplenomegaly. Neurological examination and MRI of the brain were normal. Ophthalmic pigment dilution and nystagmus were absent. Red blood cells were $2.32 \times 10^{6} / \mathrm{mm}^{3}$, hemoglobin 5.3 $\mathrm{g} / \mathrm{dl}$, platelets $12 \times 10^{3} / \mathrm{mm}^{3}$, neutrophils $1.70 \%$, monocytes $48.40 \%$. Serum chemistry profile revealed triglycerides $622 \mathrm{mg}$ /
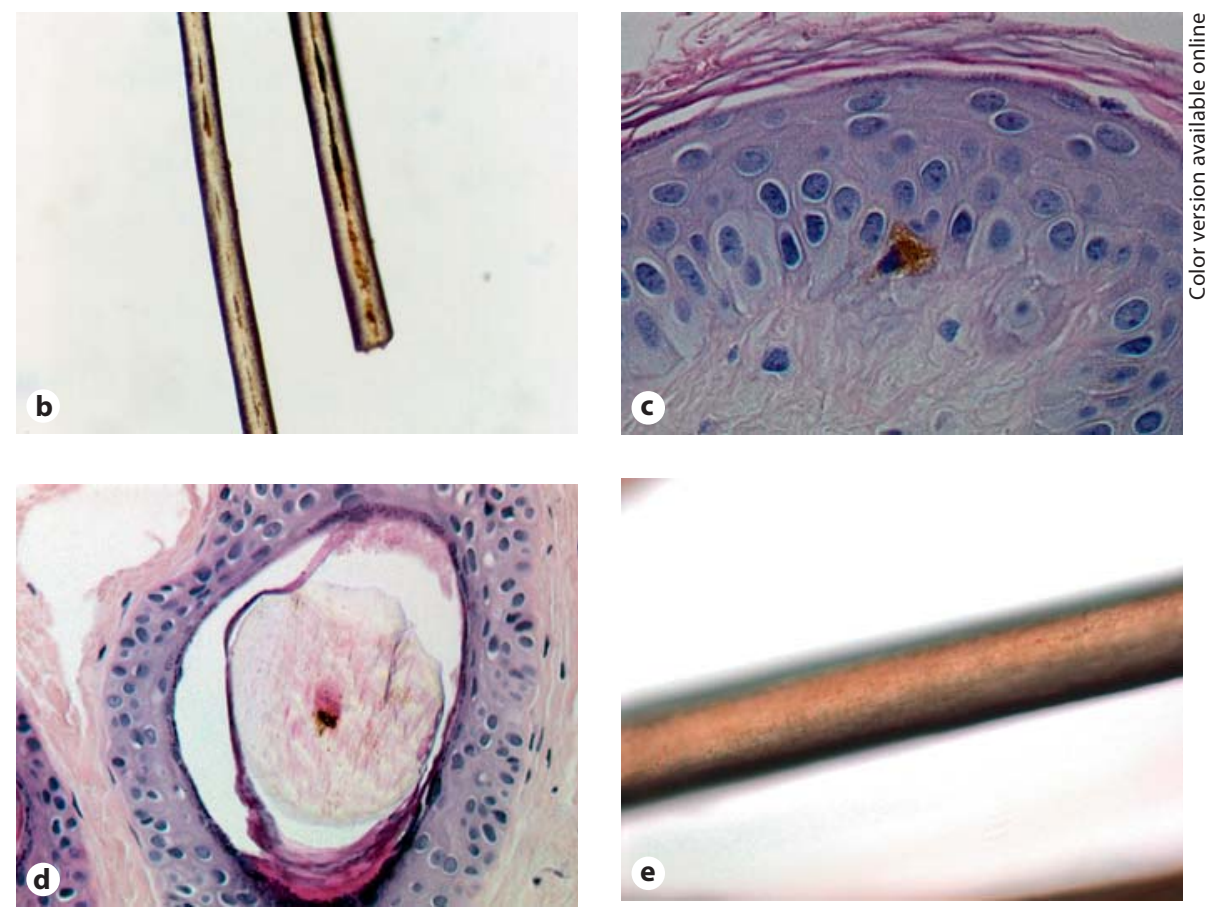

dl, fibrinogen $60 \mathrm{mg} / \mathrm{dl}, \mathrm{D}$-dimer $852 \mathrm{mg} /$ $\mathrm{ml}$, antithrombin III 141\%, aspartate aminotransferase $324 \mathrm{U} / \mathrm{l}$, alkaline phosphatase $625 \mathrm{U} / \mathrm{l}$. Bone marrow aspiration showed an increased histiocytic component. Natural killer cell function was decreased by the chromium-51 release assay at $4 \mathrm{~h}$. Repeated peripheral blood smear did not show intracytoplasmic granules in leukocytes.

Light microscopy of hair shafts revealed irregularly elongated aggregates of melanin (fig. 1b). Histological examination of a biopsy of clinically normal skin showed hyperpigmented melanocytes, with scant pigmentation in keratinocytes (fig. 1c); large clumps of melanin were also evident in the medulla of a hair shaft in a different section (fig. 1d).

Transmission electron microscopy (TEM) revealed accumulation of numerous normal-sized stage IV mature melanosomes in the cytoplasm of melanocytes; only few melanosomes were observed in adjacent keratinocytes (fig. 2). Genetic analysis, conducted at the Hôpital NeckerEnfants Malades, Paris, revealed a homozygous haplotype for the polymorphic markers linked to the RAB27A locus. Sequencing of the $R A B 27 A$ gene, performed twice, did not allow to identify any mutation.
Based on the combination of clinical features and microscopic findings, the diagnosis of GS2 was made. The patient received etoposide $\left(150 \mathrm{mg} / \mathrm{m}^{2}\right.$ a day for 3 days every 3 weeks) and methylprednisolone ( $2 \mathrm{mg} / \mathrm{kg}$ daily) to control the recurrent hemophagic seizures. In December 2001, he received allogeneic bone marrow transplantation (BMT) from a matched unrelated donor of the International Bone Marrow Donor Registry. The conditioning regimen included busulfan $4 \mathrm{mg} / \mathrm{kg}$ daily for 4 days, cyclophosphamide $60 \mathrm{mg} /$ $\mathrm{kg}$ for 2 days and rabbit antithymocyte globulin (10 mg/kg daily for 5 days). Prophylaxis for graft-versus-host disease consisted in ciclosporin A and short-course methotrexate. Infusion of $6.25 \times 10^{6} / \mathrm{kg}$ CD34+ cells was performed. Engraftment was demonstrated by the presence of $>500$ neutrophils/ $\mu$ l on day +14 and $>50 \times 10^{3}$ platelets/ $\mu$ l on day +24 . The only toxicity registered was mucositis grade 2 . He developed two infections (day +60 and +75$)$ by cytomegalovirus, treated successfully with ganciclovir and foscavir. Administration of ciclosporin A was interrupted on day +180 , without occurrence of graft-versushost disease. One year after BMT, no signs of graft-versus-host disease were observed on liver biopsy; bone marrow biopsy showed normocellularity of the three mat- 

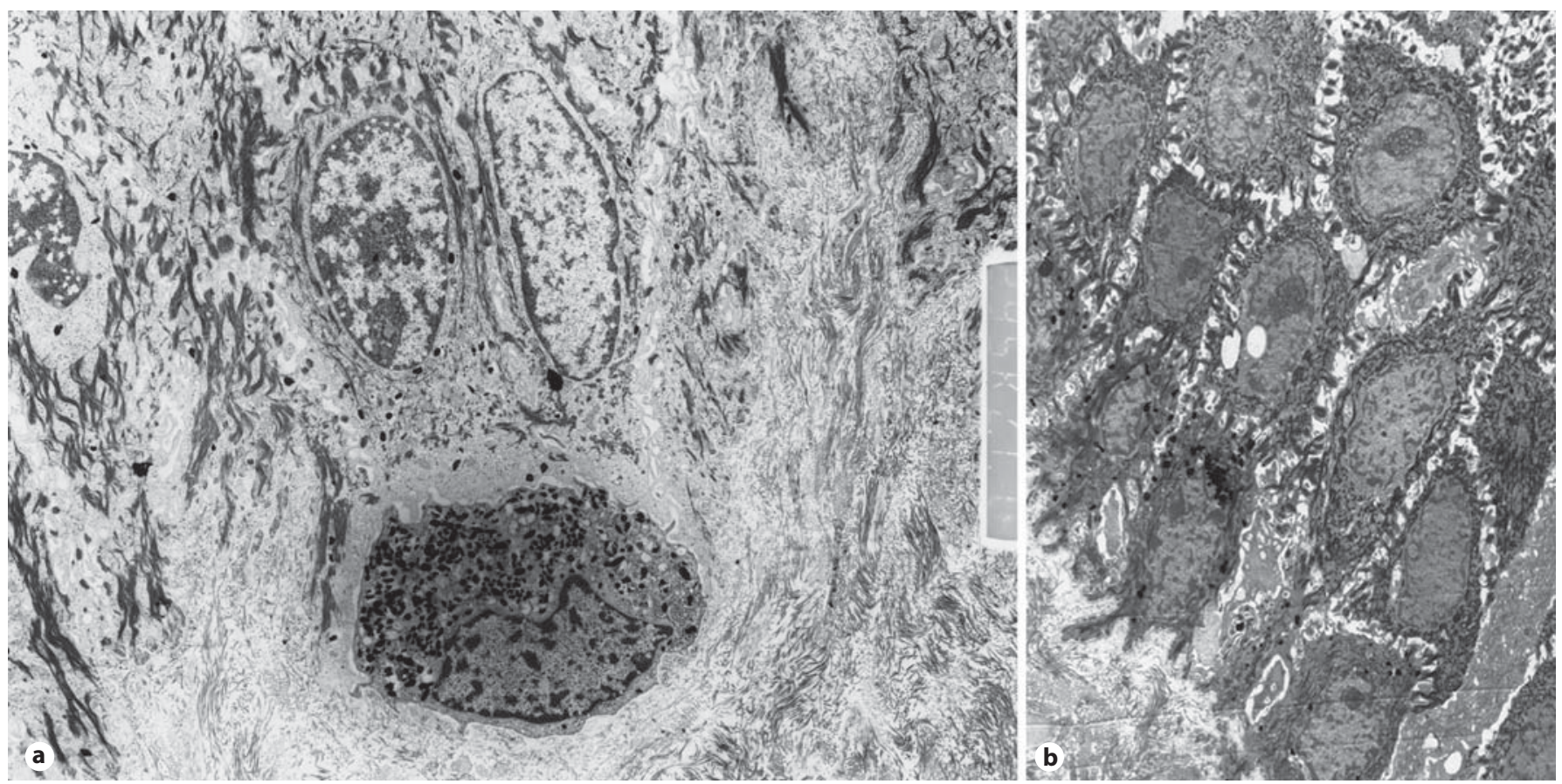

Fig. 2. a TEM showing melanocytes containing stage IV melanosomes with a perinuclear disposition. Adjacent keratinocytes contain rare and sparse melanosomes in their cytoplasm. Original magnification. $\times 3,000$. b TEM of normal epidermis of a healthy individual. Original magnification. $\times 1,900$.

uration lines. Repeated liver and bone marrow biopsies at 24 and 36 months after BMT were normal. The patient was seen 72 months after BMT, in good condition and without relapse of HLH.

\section{Discussion}

A group of inherited syndromes are characterized by silvery hair ('partial albinism') [6]. These include ES, GS and CHS. In addition to silvery hair, both GS2 and CHS display severe primary immunodeficiency. Because they are both potentially fatal and their only available cure is HSCT, early diagnosis is crucial. Currently, the diagnosis of GS2 and CHS is based on the detection of mutations of the RAB27A and the CHS1/LYST genes, respectively.

The sequencing of the RAB27A gene did not disclose any mutation in our patient. However, he was homozygous for polymorphic markers linked to the $R A$ $B 27 A$ locus. These markers have previously been described in 1 patient with GS2 [7, 8]. The most likely explanation is that our patient has GS associated with a Rab27a defect, but with a mutation in the promot- er region or inside an intron that was not detected with our approach. It would be of interest to further investigate the RAB27A gene in our patient to identify putative novel mutations.

Simple microscopic and ultrastructural features such as the absence of intracytoplasmic granules in leukocytes, the irregular distribution of large melanin clumps in the hair shaft and the presence of mature stage IV melanososmes in the cytoplasm of melanocytes and not in adjacent keratinocytes helped us in excluding CHS and establishing a diagnosis of GS. Because genetic analysis may be time-consuming and early treatment is required for GS2, rapid tests such as microscopy of hair shaft and peripheral blood smear may still prove to be valuable in the timely diagnosis of GS2.

ES/GS type 1 is also characterized by silvery hair but does not exhibit HLH [9]. In addition, nondegenerative CNS dysfunction is present. ES must be considered in the differential diagnosis of GS2 patients with cerebral lymphohistiocytic infiltration. Because in our patient the clinical signs of immunodeficiency were evident and neurologic signs absent, ES was ruled out. Moreover, in ES melanocytes are characterized by incomplete melanization of melanosomes on TEM, whereas in GS normal melanosomes accumulate in melanocytes.

The only available cure for GS2 is HSCT. Only 7 patients in the literature were found to have received allogeneic HSCT [5, 7, 8, 10-13]. To our knowledge, our patient is the second in the literature treated with BMT from an unrelated donor [13]. Although the rate of success and therefore survival is higher when BMT is performed early in life, our patient responded successfully to BMT, and we are able to document his good condition after a follow-up of 72 months. The long, uneventful follow-up of our patient supports the use of BMT from an unrelated donor if transplantation from a relative is not possible.

\section{Acknowledgment}

We are grateful to Dr. Geneviève de Saint Basile, Hôpital Necker-Enfants Malades, Paris, for genetic analysis of our patient. 


\section{References}

1 Griscelli C, Durandi A, Guy-Grand D, et al: A syndrome associating partial albinism and immunodeficiency. Am J Med 1978;65:691702 .

2 Westbroek W, Lambert J, Naeyaert JM: The dilute locus and Griscelli syndrome: gateways towards a better understanding of melanosome transport. Pigment Cell Res 2001; 14:320-327.

-3 Pastural E, Barrat FJ, Duforcq-Lagelouse R, et al: Griscelli disease maps to chromosome $15 \mathrm{q} 21$ and is associated with mutations in the myosin-Va gene. Nat Genet 1997;16:289292.

4 Blott EJ, Griffiths GM: Secretory lysosomes. Nat Rev Mol Cell Biol 2002;3:122-131.

5 Ménasché G, Ho CH, Sanal O, et al: Griscelli syndrome restricted to hypopigmentation results from a melanophilin defect (GS3) or a MYO5A F-exon deletion (GS1). J Clin Invest $2003 ; 112: 450-456$.
6 Scheinfeld NS: Syndromic albinism: a review of genetics and phenotypes. Dermatol Online J 2003;9:5.

7 Baumeister FAM, Stachel D, Schuster F, et al: Accelerated phase in partial albinism with immunodeficiency (Griscelli syndrome): genetics and stem cell transplantation in a 2month-old girl. Eur J Pediatr 2000;159:7478.

8 Schuster F, Stachel DK, Schmid I, et al: Griscelli syndrome: report of the first peripheral blood stem cell transplant and the role of mutations in the RAB27A gene as an indication for BMT. Bone Marrow Transplant 2001;28:409-412.

-9 Elejalde BR, Holguin J, Valencia A, et al: Mutations affecting pigmentation in man. 1 . Neuroectodermal melanolysosomal disease. Am J Med Genet 1979;3:65-80.
10 Klein C, Philippe N, Le Delst F, et al: Partial albinism with immunodeficiency (Griscelli syndrome). J Pediatr 1994;125:886-895.

11 Fischer A, Griscelli C, Friedrich W, et al: Bone marrow transplantation for immunodeficiencies and osteopetrosis: European survey 1968-1985. Lancet 1986;ii:10801083.

12 Schneider LC, Berman RS, Shea CR, et al: Bone marrow transplantation for the syndrome of pigmentary dilution and lymphohistiocytosis (Griscelli's syndrome). J Clin Immunol 1990;10:146-153.

13 Aricò M, Zecca M, Santoro N, et al: Successful treatment of Griscelli syndrome with unrelated donor allogeneic hematopoietic stem cell transplantation. Bone Marrow Transplant 2002;29:995-998. 